\title{
Efficacy and Safety of HLX03, an Adalimumab Biosimilar, in Patients with Moderate-to-Severe Plaque Psoriasis: A Randomized, Double-Blind, Phase III Study
}

Lin Cai · Linfeng Li $\cdot$ Hao Cheng $\cdot$ Yangfeng Ding $\cdot$ Zhenshu Biao •

Shifa Zhang · Songmei Geng · Quanzhong Liu · Hong Fang ·

Zhiqi Song $\cdot$ Yan $\mathrm{Lu} \cdot$ Shanshan $\mathrm{Li} \cdot$ Qing Guo $\cdot$ Juan Tao $\cdot$

Li He · Jun Gu · Qinping Yang · Xiuping Han · Xinghua Gao ·

Danqi Deng $\cdot$ Shenqiu Li $\cdot$ Qingyu Wang $\cdot$ Jun Zhu $\cdot$ Jianzhong Zhang

Received: July 15, 2021 / Accepted: August 19, 2021 / Published online: November 23, 2021

(c) The Author(s) 2021

\section{ABSTRACT}

Introduction: Adalimumab has been used successfully in the treatment of psoriasis. The objective of the study is to compare the efficacy,

Supplementary Information The online version contains supplementary material available at https://doi. org/10.1007/s12325-021-01899-0.

L. Cai · J. Zhang (ه)

Department of Dermatology, Peking University People's Hospital, No. 11 Xizhimen South Avenue, Beijing 100044, China

e-mail: rmzjz@126.com

L. Li

Department of Dermatology, Beijing Friendship

Hospital, Capital Medical University, Beijing, China

H. Cheng

Department of Dermatology, Sir Run Run Shaw

Hospital, School of Medicine, Zhejiang University,

Hangzhou, China

Y. Ding

Department of Dermatology, Shanghai Skin Disease

Hospital, Shanghai, China

Z. Biao

Department of Dermatology, Liaoning Provincial

People's Hospital, Shenyang, China

S. Zhang

Department of Dermatology, General Hospital of

Shenyang Military Region, Shenyang, China safety, and immunogenicity between HLX03, an adalimumab biosimilar, and adalimumab in Chinese patients with moderate-to-severe plaque psoriasis.

Methods: In this double-blind, active-controlled, parallel-group study, 262 patients with moderate-to-severe plaque psoriasis were randomized (1:1) to receive HLX03 or adalimumab (80 $\mathrm{mg}$ at week $1,40 \mathrm{mg}$ at week 2 , and then

\section{S. Geng}

Department of Dermatology, The Second Affiliated Hospital of Xi'an Jiaotong University, Xi'an, China

Q. Liu

Department of Dermatology, Tianjin Medical University General Hospital, Tianjin, China

\section{H. Fang}

Department of Dermatology, The First Affiliated Hospital of Zhejiang University College of Medicine, Hangzhou, China

\section{Z. Song}

Department of Dermatology, The First Affiliated Hospital of Dalian Medical University, Dalian, China

Y. Lu

Department of Dermatology, Jiangsu Provincial People's Hospital, Nanjing, China

S. Li

Department of Dermatology, The First Hospital of Jilin University, Changchun, China 
$40 \mathrm{mg}$ every 2 weeks) for 48 weeks. The primary endpoint was improvement in Psoriasis Area and Severity Index (PASI) score at week 16 comparing to baseline. Equivalence was demonstrated if 95\% confidence interval (CI) of the between group difference fell within the equivalence margins of $\pm 15 \%$. Other efficacy endpoints, safety and immunogenicity were also evaluated.

Results: In the full analysis set, PASI improvements at week 16 was $83.5 \%(n=131)$ in the HLX03 group and $82.0 \%(n=130)$ in the adalimumab group, with a least-square-mean difference of $1.5 \%$ (95\% CI $-3.9 \%$ to $6.8 \%$ ). There were no significant between-group differences in all secondary efficacy analyses including proportion of patients achieving $\geq 75 \%$ improvement from baseline PASI (PASI 75), physician global assessment (PGA) 0/1 (clear or almost clear) and change in dermatology life quality index (DLQI) score. The incidences of adverse events and the proportion of patients with antidrug antibodies were also comparable between the two treatment groups.

Conclusion: HLX03 demonstrated equivalent efficacy, similar safety and immunogenicity to reference adalimumab, supporting its development as an alternative treatment for patients with plaque psoriasis in China.

Clinical trial registration: Chinadrugtrials.org.cn, CTR20171123 (November 27, 2017);

Q. Guo

Department of Dermatology, Sun Yat-Sen Memorial

Hospital, Sun Yat-Sen University, Guangzhou, China

J. Tao

Department of Dermatology, Union Hospital, Tongji Medical College, Huazhong University of Science and Technology, Wuhan, China

L. He

Department of Dermatology, The First Affiliated Hospital of Kunming Medical University, Kunming, China

J. Gu

Department of Dermatology, Changhai Hospital, Shanghai, China

Q. Yang

Department of Dermatology, Huashan Hospital, Fudan University, Shanghai, China
ClinicalTrials.gov, NCT03316781 (October 20, 2017).

\section{PLAIN LANGUAGE SUMMARY}

Plaque psoriasis is a chronic, autoimmune, inflammatory skin disease associated with significant morbidity and reduced quality of life. In China, the prevalence of plaque psoriasis increased four-fold between 1987 and 2012. Adalimumab is a biologic antibody used to treat plaque psoriasis globally. However, high treatment costs remain as a significant barrier to adalimumab therapy. Therefore, HLX03 has been developed as an adalimumab (Humira ${ }^{\circledR}$ ) biosimilar, which is almost identical to the licensed reference adalimumab, but less expensive and more accessible to patients. In this randomized clinical trial, the efficacy (ability of a drug to produce the desired treatment effects), safety, and immunogenicity (ability of a drug to induce immune response which would affect its efficacy and safety) of HLX03 were compared with the reference adalimumab in Chinese patients with moderate-to-severe plaque psoriasis. Efficacy was evaluated by comparing the changes in severity and extent of disease using Psoriasis Area and Severity Index score between treatment initiation and week 16 . Safety was monitored by adverse events, laboratory tests

\section{Han}

Department of Dermatology, Shengjing Hospital, China Medical University, Shenyang, China

X. Gao

Department of Dermatology, The First Hospital of China Medical University, Shenyang, China

D. Deng

Department of Dermatology, The Second Affiliated Hospital of Kunming Medical University, Kunming, China

S. Li

Department of Dermatology, Tongji Hospital, Tongji Medical College, Huazhong University of Science and Technology, Wuhan, China

Q. Wang · J. Zhu

Shanghai Henlius Biotech, Inc., Shanghai, China 
and vital signs. Immunogenicity was assessed by the incidence of antidrug antibodies. Among the 262 randomized patients, 131 received HLX03 and 130 received adalimumab. Both groups reported similar improvements in Psoriasis Area and Severity Index scores (betweengroup difference fell within the prespecified equivalence margins), and also in other efficacy evaluations. Additionally, the two treatment groups showed similar safety and immunogenicity profiles. In summary, HLX03 demonstrated equivalent efficacy to adalimumab, validating it as an alternative treatment for patients with plaque psoriasis in China.

Keywords: Adalimumab; Biosimilar; Plaque psoriasis; $\mathrm{TNF} \alpha$ inhibitor

\section{Key Summary Points}

This phase III equivalence study aimed to evaluate the clinical equivalence between HLX03 (an adalimumab biosimilar) and the reference adalimumab in patients with moderate-to-severe plaque psoriasis.

HLX03 and adalimumab showed equivalence efficacy in moderate-to-severe plaque psoriasis patients.

Similar safety and immunogenicity profiles were observed between HLX03 and adalimumab.

As an approved biosimilar to adalimumab, HLX03 provides an alternative treatment option for patients with moderate-tosevere plaque psoriasis.

\section{INTRODUCTION}

Psoriasis is a chronic, immune-mediated, inflammatory disease affecting the skin, nails, and joints and is associated with significant morbidity and reduced quality of life. In 2012, the reported prevalence of psoriasis in China was $0.47 \%$, representing a four-fold increase over 25 years [1].

Adalimumab (Humira ${ }^{\circledR}$, AbbVie, IL, USA) is a recombinant humanized monoclonal anti-tumor necrosis factor alpha (anti-TNF $\alpha$ ) antibody which has been approved globally for various immune-mediated conditions [2, 3], and in China for the treatment of rheumatoid arthritis, ankylosing spondylitis, Crohn's disease, and plaque psoriasis $[4,5]$. However, there remains an unmet need for more cost-effective treatment options [6, 7].

Biosimilars are highly similar to the licensed biological products, but are less expensive and more accessible to patients [8]. Before a biosimilar can be authorized in any regulated country, similarity must be established between the biosimilar and its originator product in quality, biological activity, structure/physicochemical and functional attributes, efficacy, safety, and immunogenicity [9-13]. Several adalimumab biosimilars have been approved for the treatment of inflammatory diseases by the United States Food and Drug Administration and European Medicines Agency, and four adalimumab biosimilars (BAT1406, IBI303, HS016, and HLX03) have been approved by China National Medical Products Administration (NMPA) [14-18]. Among these biologics, the confirmatory phase III studies of BAT1406, IBI303, and HS016 were conducted in patients with ankylosing spondylitis. HLX03 (Shanghai Henlius Biotech, China) is the first NMPA-approved adalimumab biosimilar (December 2, 2020) [19] evaluated in patients with moderateto-severe plaque psoriasis.

HLX03 possesses the same amino acid sequence, and has comparative structural, functional, and preclinical characteristics to adalimumab [20]. Moreover, in a phase I study among healthy adult Chinese males, similar pharmacokinetic (PK) and safety profiles were observed between HLX03 and adalimumab [21]. Here, we report the phase III study, which was designed to validate the efficacy and safety similarity between HLX03 and adalimumab in patients with moderate-to-severe plaque psoriasis in China. 


\section{METHODS}

\section{Study Design}

This was a randomized, double-blind, activecontrolled, parallel-group study carried out between 24 October 2017 and 29 March 2019 at 21 centers in China [Electronic Supplementary Material (ESM) Table S1]. The primary objective was to compare the efficacy of HLX03 and adalimumab in Chinese patients with moderate-to-severe plaque psoriasis. Secondary objectives were to evaluate the safety, tolerability, and immunogenicity of HLX03 and adalimumab.

A central interactive web response system was used to assign patients 1:1 to receive HLX03 or adalimumab with stratification of prior biological use (yes/no) according to their unique randomization code. Patients received an initial loading dose of $80 \mathrm{mg}$ study drug on day 1 of week 1 , followed by $40 \mathrm{mg}$ on day 1 of week 2 and $40 \mathrm{mg}$ every other week thereafter until week 48. Patients were followed up for 4 weeks. An end-of-treatment efficacy visit was performed at week 50, and an end-of-treatment safety visit was performed at week 52 . Patients, the investigators, and the study sponsor remained blinded to treatment allocations. Study drugs were administered via subcutaneous injection by an appointed 'unblinded' nurse.

The trial was designed according to the Guidelines for Development and Evaluation of Biosimilar Drugs issued by the NMPA and was performed in accordance with Good Clinical Practice and the Declaration of Helsinki. The study protocol and all amendments were reviewed by independent ethics committees at each center, and all patients provided written informed consent.

\section{Study Population}

Adults aged 18-75 years with moderate-to-severe plaque psoriasis were eligible if they had stable disease for $\geq 6$ months; were able to receive systemic therapy but had shown inadequate response or treatment intolerance, contraindication, or failure; had $\geq 10 \%$ body surface area (BSA) affected by psoriasis, a Physician Global Assessment (PGA) score $\geq 3$ and a Psoriasis Area and Severity Index (PASI) score $\geq 12$. Patients were excluded if they had a history of tuberculosis or active tuberculosis, nonplaque or drug-induced psoriasis, or any other skin conditions or systemic inflammatory diseases that might interfere with the evaluation of treatment efficacy. Patients who had used etanercept (within 4 weeks) or other biological agents (within 12 weeks), or who received trial-related drugs within 3 months prior to screening were also excluded. Full inclusion/exclusion criteria are listed in ESM Appendix S1.

\section{Assessments}

The primary efficacy endpoint was the percentage improvement in PASI score at week 16 comparing to baseline. Secondary efficacy endpoints included the percentage of patients who achieved a $75 \%$ improvement in PASI score (PASI 75), the percentage of patients who achieved a PGA 0/1 (clear or almost clear), and the change in Dermatology Life Quality Index (DLQI) score relative to baseline at weeks 4,8 , $12,16,20,32$, and 50 . A change in DLQI score of five points was considered significant, and a change of $\leq 1$ point was considered as no or little effect.

Safety was assessed up to week 52 by monitoring treatment-emergent adverse events (TEAEs), serious adverse events (SAEs), laboratory tests, and vital signs. Adverse events of special interest (AESIs) including infections, malignancies, hepatobiliary abnormalities in laboratory tests, and injection site reactions which were previously reported in patients treated with adalimumab or other TNF blockers [2] were also described in this study.

Plasma samples for evaluating serum drug concentrations and immunogenicity were collected at baseline and at weeks 4, 16, 20, 32, and 52. A bridging electrochemiluminescence assay was used to detect the presence of antidrug antibodies (ADAs) and a validated method based on an L-929 cell proliferation endpoint 
was used to measure neutralizing ADAs (NADAs). Both tests were conducted at a central laboratory (United-Power Pharma Tech, Beijing, China). The assay sensitivities for ADAs and NADAs were approximately $5.2 \mathrm{ng} / \mathrm{mL}$ and $187.5 \mathrm{ng} / \mathrm{mL}$, respectively.

\section{Statistical Analysis}

Following the methodology of a previous study [22] and technical guidance from the NMPA, a sample size of 108 patients per group was determined to give an $80 \%$ power to achieve a statistical significance of 0.05 using a two-sided test for comparison of HLX03 and adalimumab, with a dropout rate of $20 \%[4,9]$.

Efficacy data were analyzed using the full analysis set (FAS; all randomized patients receiving $\geq 1$ dose of study drug and $\geq 1$ postdose efficacy evaluation) and the per-protocol set (PPS; all FAS patients without major protocol violations).

The primary efficacy endpoint was analyzed using the analysis of covariance model (ANCOVA), with treatment group as a fixed effect and previous use of biological therapy (yes/no) and baseline PASI as covariates to calculate the adjusted means, standard errors, and between-treatment differences. Equivalence was determined if the 95\% confidence interval (CI) of the between-group difference fell within the predefined range of $\pm 15 \%$. Subgroup analyses of the primary endpoint were performed on the basis of following factors: age ( $<65$ years old, $\geq 65$ years old); gender (male, female); duration of psoriasis ( $<$ median, $\geq$ median); and previous use of biological therapy (yes/no), nonbiological therapy (yes/no), or traditional diseasemodifying antirheumatic drugs (yes/no).

Secondary efficacy endpoints were analyzed using a mixed-effects model (for repeated measures) and a covariance analysis model. For continuous variables (percentage improvement of PASI and change in DLQI), adjusted means and standard errors of the change (or percentage) relative to baseline and two-sided 95\% CIs were calculated. For categorical variables (PASI 75 and PGA 0/1), response rates, standard errors, odds ratios, and two-sided 95\% CIs were calculated.

Safety data were analyzed using the safety set (all patients receiving $\geq 1$ dose of study drug who had post-dose safety data). Immunogenicity analysis included patients in the safety set who had at least one evaluable antibody test result. PK data were analyzed using the PK full analysis set, which included all randomized subjects with at least one PK parameter after receiving the study drugs.

All statistical analyses were performed using SAS v.9.4 software (SAS Institute, Cary, NC, USA).

\section{RESULTS}

\section{Patient Baseline Demographics and Characteristics}

Of the 262 randomized patients, 261 received $\geq 1$ dose of the study drug (HLX03, $n=131$; adalimumab, $n=130$ ) and 214 completed the study (HLX03, $n=108$; adalimumab, $n=106$ ) (Fig. 1). The most common reasons for early withdrawal were TEAE and withdrawal of informed consent.

Baseline characteristics were well balanced between the two groups (Table 1) with the exception of higher baseline median PASI scores and median BSA affected by psoriasis in the HLX03 group compared with those in the adalimumab group (24.3 vs. 20.4 and 40.0 vs. 35.3 , respectively).

\section{Primary Efficacy Endpoint}

In the FAS, the percentage improvements in PASI at week 16 were $83.5 \%$ and $82.0 \%$ in the HLX03 and adalimumab groups, respectively (Fig. 2). The least-square-mean difference was $1.5 \%$ with a $95 \%$ CI of $-3.9 \%$ to $6.8 \%$, which fell within the predefined equivalence margins $( \pm 15 \%)$. The primary endpoint was also equivalent in the PPS $[85.1 \%$ vs. $84.5 \%$, respectively, with a between-group difference of $0.6 \%(95 \% \mathrm{CI}-3.3 \%$ to $4.6 \%)]$. 


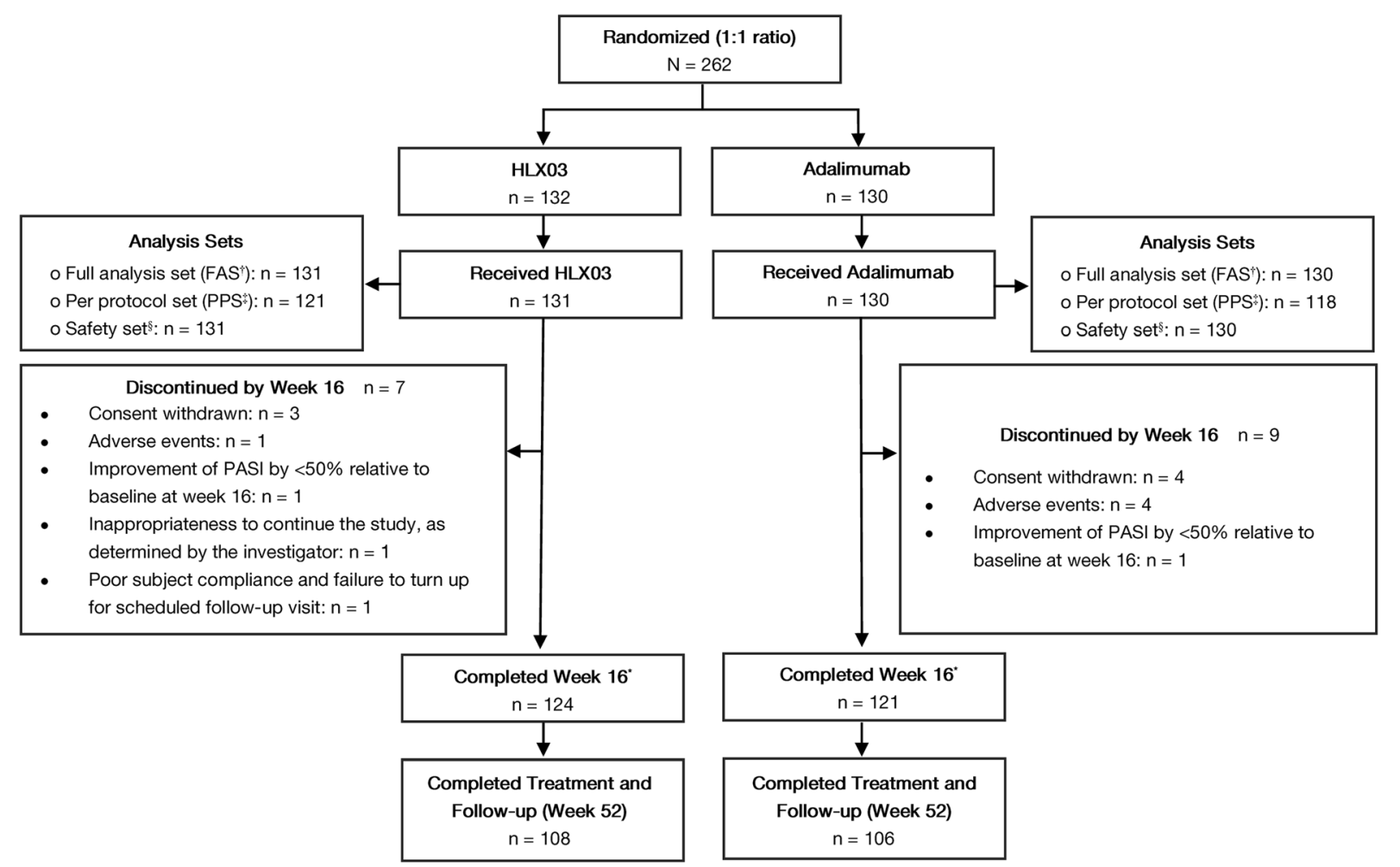

Fig. 1 Patient disposition. "Time of primary efficacy endpoint: percentage improvement in PASI score from baseline to Week 16. ${ }^{\dagger}$ FAS includes all randomized patients who received at least one dose of the study drug and had baseline values and at least one post-dose efficacy evaluation. "PPS includes all patients in the FAS who had no major protocol violations. Ten treated patients were excluded from the PPS in the HLX03 group (seven patients with $<16$ weeks of dosing, two patients for not receiving study treatment at some visits and one patient for use of prohibited drugs). Twelve treated patients were

Persistent improvements in PASI were observed at weeks $4,8,12,20,32$, and 50, with no significant between-group differences for either the whole FAS (Fig. 2) or any subgroups (ESM Fig. S1).

\section{Secondary Efficacy Endpoints}

At week 16, PASI 75 response rates were $92.0 \%$ in the HLX03 group and $88.7 \%$ in the adalimumab group [relative risk (RR): 1.1 (95\% CI 0.8 to 1.4)]. PASI 75 response rates were sustained in $>90 \%$ of patients until week 50 , and there were no statistical differences between groups at

excluded from the PPS in the adalimumab group (eight patients with $<16$ weeks of dosing, one patient for not receiving study treatment at some visits, two patients for use of prohibited drugs and one patient erroneously injected with the drug meant for other patients). ${ }^{\$}$ Safety population includes all patients who received at least one dose of the study drug and had post-dose safety evaluation data. FAS full analysis set, PASI Psoriasis Area and Severity Index, PPS per protocol set

any visit (Fig. 3a). The percentage of patients reaching PGA 0/1 improved persistently until week 50, with no significant differences between treatments except at week 8 , when the percentage in the HLX03 group was lower [RR 0.5 (95\% CI 0.3-0.9)] (Fig. 3b). From week 4, DLQI improved significantly at each time point in both groups, with no significant betweengroup differences (Fig. 4). Results of secondary efficacy endpoints in the PPS were consistent with those in the FAS (data not shown). 
Table 1 Baseline demographics and characteristics (FAS)

\begin{tabular}{lll}
\hline Characteristic & $\begin{array}{l}\text { HLX03 } \\
\boldsymbol{n}=\mathbf{1 3 1}\end{array}$ & $\begin{array}{l}\text { Adalimumab } \\
\boldsymbol{n}=\mathbf{1 3 0}\end{array}$ \\
\hline Age, median (range), years & $38.0(18.0-74.0)$ & $38.5(19.0-71.0)$ \\
Male, $n$ (\%) & $99(75.6)$ & $91(70.0)$ \\
Han ethnicity, $n$ (\%) & $130(99.2)$ & $126(96.9)$ \\
Weight, median (range), kg & $74.0(47.0-112.0)$ & $74.0(44.0-167.0)$ \\
Duration of psoriasis, median years & 12.7 & 13.4 \\
PASI score, median (Q1, Q3) & $24.3(18.0-32.9)$ & $20.4(14.9-26.1)$ \\
BSA affected, median (Q1, Q3) & $40.0(29.0-59.0)$ & $35.3(22.4-49.0)$ \\
PGA, $n$ (\%) & & \\
Clear/almost clear & 0 & 0 \\
Moderate & 69 & 94 \\
Severe & 62 & 36 \\
DLQI score, median (Q1, Q3) & $15.0(8.0-21.0)$ & $15.0(10.0-22.0)$ \\
Previous psoriasis medication, $n(\%)$ & $131(100)$ & $130(100)$ \\
Biological therapy & $11(8.4)$ & $9(6.9)$ \\
Concomitant psoriasis medication, $n(\%)$ & $72(55.0)$ & $73(56.2)$ \\
Traditional/herbal medicine & $51(38.9)$ & $52(40.0)$ \\
Nonbiological anti-psoriatic drugs & $33(25.2)$ & $37(28.5)$ \\
Glucocorticoids & $23(17.6)$ & $29(22.3)$ \\
\hline BS & & \\
\hline
\end{tabular}

$B S A$ body surface area, $D L Q I$ Dermatology Life Quality Index, FAS full analysis set, $P A S I$ Psoriasis Area and Severity Index, $P G A$ Physician Global Assessment, $Q$ quartile

\section{Safety}

The overall safety profiles of HLX03 and adalimumab were similar. The incidence of TEAEs was $89.3 \%(117 / 131)$ in the HLX03 group and $94.6 \%(123 / 130)$ in the adalimumab group, with no significant differences between groups in the incidence of AESIs, SAEs, or TEAEs leading to drug discontinuation (Table 2). The most common TEAEs were upper respiratory tract infection, hyperuricemia, liver dysfunction and urinary tract infection (Table 2). Most TEAEs were mild in severity $[85 / 117(72.6 \%)$ for HLX03 and 86/123 (69.9\%) for adalimumab]. Seven (5.3\%) HLX03-treated patients experienced 15 SAEs and nine (6.9\%) adalimumab-treated patients experienced 10 SAEs. The most common SAE in both groups was tuberculosis, with three active and two suspected cases in the HLX03 and adalimumab groups and adalimumab groups, respectively.

The incidence of AESIs was similar between two groups: 87 patients (66.4\%) in the HLX03 group developed 203 AESIs and 92 patients $(70.8 \%)$ in the adalimumab group developed 253 AESIs. The most common AESIs were upper respiratory tract infection and liver dysfunction (Table 2). Most AESIs were mild [73/87 (83.9\%) and 74/92 (80.4\%) patients in the HLX03 and adalimumab groups, respectively], including one patient tested positive for hepatitis B core 


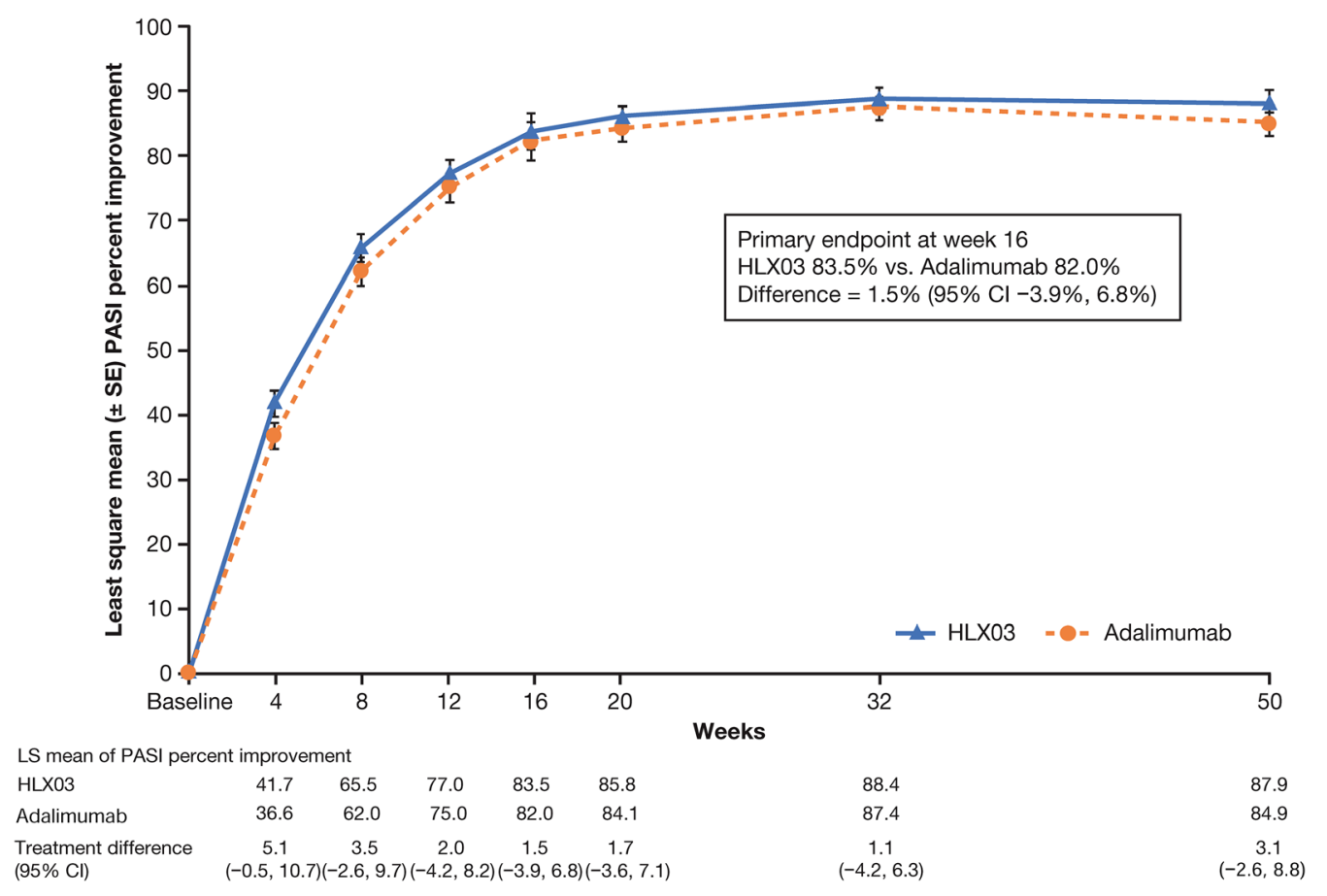

Fig. 2 Percentage improvement in PASI over time through week 50 (FAS). FAS full analysis set, LS least square, PASI Psoriasis Area and Severity Index, $S E$ standard error

antibody in the adalimumab group. One patient in the adalimumab group experienced a severe AESI (iridocyclitis). No new malignancies or deaths occurred, and there were no clinically significant abnormalities in laboratory test results, vital signs, physical examinations, or electrocardiogram test results.

\section{Immunogenicity}

The proportion of patients with ADAs increased gradually with the administration of study drugs and did not differ significantly between treatment groups at any time (Table 3). The percentage of patients with at least one positive test for ADAs was $81.9 \%$ in the HLX03 group and $80.6 \%$ in the adalimumab group. The accumulated proportions of patients with ADAs at week 52 were $69.5 \%$ and $73.7 \%$, respectively (Table 3).

The proportion of patients with NADAs had no significant association with duration of drug administration. The accumulated proportions of patients with NADAs at week 52 were $17.1 \%$ (HLX03) and 21.4\% (adalimumab) (Table 3).

\section{DISCUSSION}

Prior to HLX03, phase III studies of all NMPAapproved adalimumab biosimilars were conducted in patients with ankylosing spondylitis [16-18]. Although indication extrapolation avoids unnecessary conduction of clinical trials, some societies are concerned, as limited clinical efficacy and safety data are available for the extrapolated indications, which potentially restrict therapeutic uptake [23]. The study reported here is the first phase III study of adalimumab biosimilars conducted in patients with plaque psoriasis in China. Psoriasis represents a more sensitive indication for tests of clinical similarity with a relatively pronounced effect size by monotherapy when compared to other inflammatory diseases [24, 25]. 
(a)

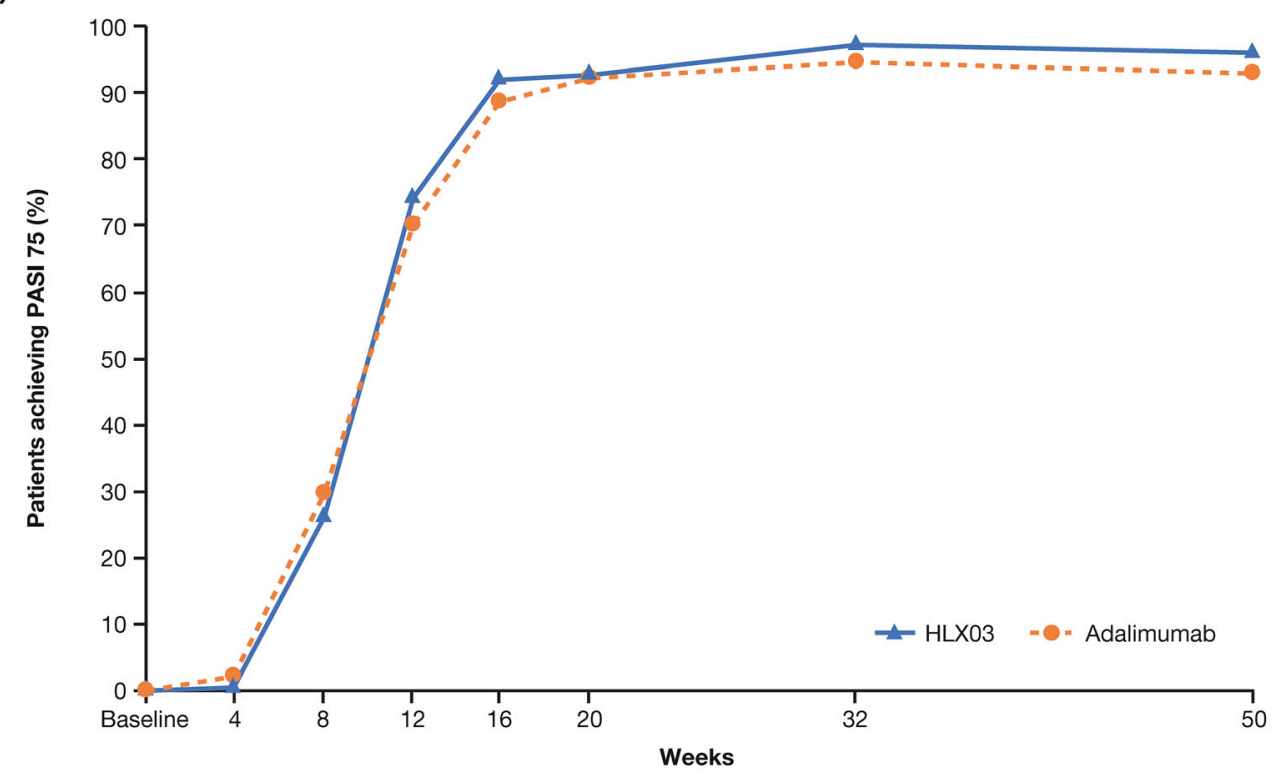

Response rate (\%)

$\begin{array}{lccccc}\text { HLX03 } & 0.5 & 26.0 & 74.2 & 92.0 & 92.7 \\ \text { Adalimumab } & 2.2 & 29.6 & 70.1 & 88.7 & 92.3 \\ \text { Relative risk } & 0.3 & 0.9 & 1.1 & 1.1 & 1.0 \\ (95 \% \mathrm{Cl}) & (0.1,1.2) & (0.6,1.4) & (0.8,1.4) & (0.8,1.4) & (0.8,1.3)\end{array}$

97.2

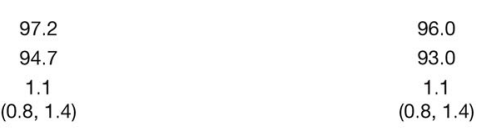

(b)

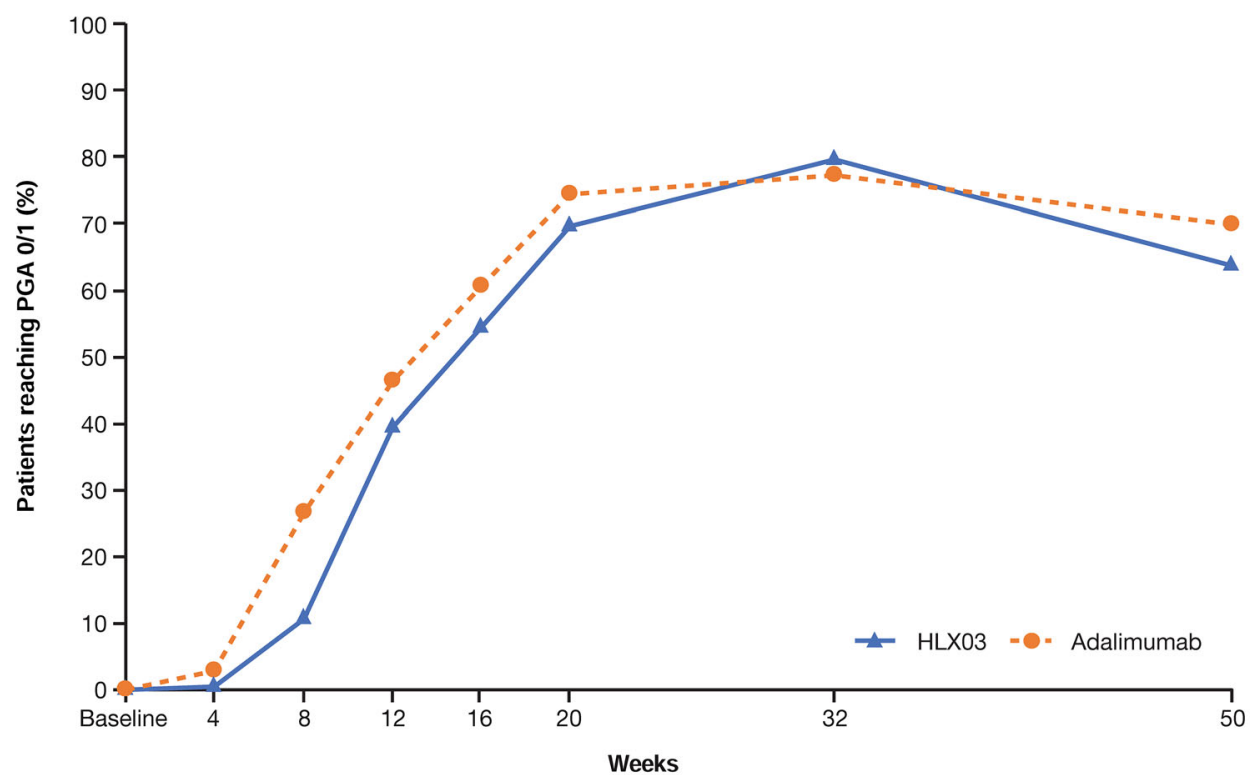

Response rate (\%)

HLX03

Adalimumab

Relative risk

$(95 \% \mathrm{Cl})$

$\begin{array}{ccccc}0.4 & 10.6 & 39.5 & 54.4 & 69.5 \\ 2.8 & 26.6 & 46.2 & 60.5 & 74.3 \\ 0.2 & 0.5 & 0.9 & 0.9 & 0.9 \\ (0.0,1.4) & (0.3,0.9) & (0.6,1.3) & (0.7,1.3) & (0.7,1.3)\end{array}$

$(0.0,1.4)(0.3,0.9)(0.6,1.3)(0.7,1.3)(0.7,1.3)$
79.6
77.3
1.0

$(0.8,1.4)$
$(0.8,1.4)$ 
4 Fig. 3 Percentage of patients achieving a PASI 75 and b PGA $0 / 1$ over time through week 50 (FAS). FAS full analysis set, $P A S I 75$ an improvement in Psoriasis Area and Severity Index of at least $75 \%$ relative to baseline, $P G A 0 / 1$ Physician's Global Assessment of clear or almost clear

In this study, HLX03 demonstrated equivalent efficacy to adalimumab based on the primary endpoint of percentage improvement in PASI score at week 16, as well as in subgroup analyses stratified by age, sex, duration of psoriasis, and previously used therapy. Equivalence was also demonstrated in the analyses of secondary efficacy endpoints (PASI 75, PGA 0/1, and DLQI). As improved quality of life is an important goal of biological therapy, it is noteworthy that patients treated with HLX03 or adalimumab showed significant and persistent improvements in DLQI over 50 weeks, starting as early as week 4 .
The primary endpoint was chosen because it is a continuous variable and may offer a better comparative analysis than a binary assessment of meeting a minimum response threshold [22]. It also reflects current treatment goals that emphasize improvements in the severity and extent of psoriasis [26]. The efficacy results were broadly comparable with those from earlier trials investigating adalimumab treatment in Chinese [27] European [28] North American [29], or Japanese [30] patients with plaque psoriasis.

HLX03 and adalimumab showed comparable safety profiles up to week 52 : the incidence and type of TEAEs were generally consistent with the known safety profile of adalimumab in patients with plaque psoriasis $[2,27]$. Similar to a recent trial assessing adalimumab in Chinese patients with plaque psoriasis, upper respiratory tract infection was the most common AESI [27]. In the current trial, most AESIs were mild with only one patient treated with adalimumab

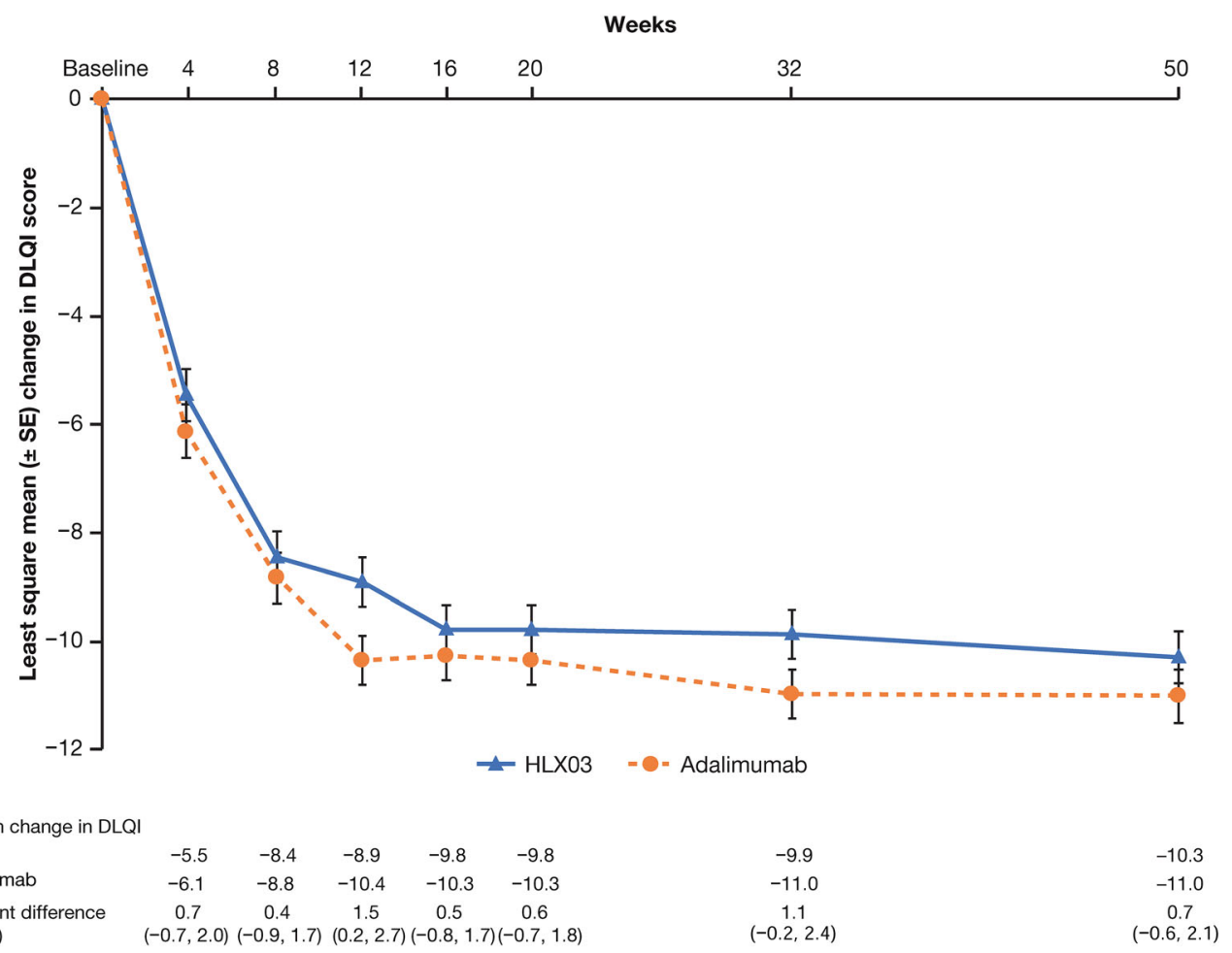

Fig. 4 Change in DLQI over time through week 50 (FAS). DLQI Dermatology Life Quality Index, FAS full analysis set, LS least square, $S E$ standard error 
Table 2 Safety summary: TEAEs up to week 52 (safety set)

\begin{tabular}{|c|c|c|}
\hline Number of patients (\%) & $\begin{array}{l}\text { HLX03 } \\
n=131\end{array}$ & $\begin{array}{l}\text { Adalimumab } \\
n=130\end{array}$ \\
\hline TEAEs & $117(89.3)$ & $123(94.6)$ \\
\hline SAEs & $7(5.3)$ & $9(6.9)$ \\
\hline AESIs $^{a}$ & $87(66.4)$ & $92(70.8)$ \\
\hline TEAEs leading to study discontinuation & $9(6.9)$ & $7(5.4)$ \\
\hline Deaths & 0 & 0 \\
\hline \multicolumn{3}{|c|}{ TEAEs with an incidence $\geq 10 \%$ in any group } \\
\hline Upper respiratory infection & $37(28.2)$ & $34(26.2)$ \\
\hline Hyperuricaemia & $24(18.3)$ & $18(13.9)$ \\
\hline Liver dysfunction ${ }^{\mathrm{b}}$ & $23(17.6)$ & $22(16.9)$ \\
\hline Urinary tract infection & $16(12.2)$ & $12(9.2)$ \\
\hline Hyperlipidaemia & $10(7.6)$ & $16(12.3)$ \\
\hline Nasopharyngitis & $10(7.6)$ & $13(10.0)$ \\
\hline \multicolumn{3}{|c|}{ AESIs by system organ class with an incidence $\geq 3 \%$ in any group ${ }^{a}$} \\
\hline Infections and infectious diseases & $62(47.3)$ & $60(46.2)$ \\
\hline Upper respiratory infection & $37(28.2)$ & $30(23.1)$ \\
\hline Urinary tract infection & $10(7.6)$ & $10(7.7)$ \\
\hline Hepatobiliary disorders & $26(19.9)$ & $27(20.8)$ \\
\hline Liver dysfunction & $23(17.6)$ & $22(16.9)$ \\
\hline
\end{tabular}

AESI adverse event of special interest, $S A E$ serious adverse event, TEAE treatment-emergent adverse event

a AESIs in this study included: tolerability at injection site, hepatobiliary abnormalities in laboratory test, infections (including tuberculosis), new active tuberculosis and new malignancy

${ }^{\mathrm{b}}$ One patient in the adalimumab group withdrew from the study due to reported liver function abnormalities; the patient was hospitalized and the subsequent discharge diagnosis was updated to acute hepatitis B

experienced a severe AESI of iridocyclitis. Three active (in the HLX03 group) and two suspected (in the adalimumab group) cases of tuberculosis were possibly related to the study drugs, but all recovered (or were recovering) by the end of the study. Importantly, given the potential association between TNF $\alpha$ inhibitors and an increased risk of serious infections and cancers [31, 32], no new safety signals, malignancies or death were observed in this study.

Immunogenicity was similar between HLX03 and adalimumab at all time points, and ADA positive rates increased gradually with drug administration. The trends are similar to other head-to-head trials of adalimumab biosimilars (vs. the reference compound) [33]. The high positive rates of ADA were similar to those reported in recent studies of adalimumab biosimilars, in which assays with higher sensitivity were used $[17,34,35]$. In addition to detection methods, previous medication history and ethnicity might also contribute to the high positive rates of ADA in this study $[17,36]$.

Like all randomized controlled trials, generalizability of the results may be limited by a patient population that is less heterogeneous 
Table 3 Immunogenicity following treatment of HLX03 and adalimumab

\begin{tabular}{|c|c|c|c|c|}
\hline & \multicolumn{2}{|l|}{ ADAs } & \multicolumn{2}{|l|}{ NADAs } \\
\hline & $\begin{array}{l}\text { HLX03 } \\
n / N(\%)\end{array}$ & $\begin{array}{l}\text { Adalimumab } \\
n / N(\%)\end{array}$ & $\begin{array}{l}\text { HLX03 } \\
n / N(\%)\end{array}$ & $\begin{array}{l}\text { Adalimumab } \\
n / N(\%)\end{array}$ \\
\hline Baseline & $4 / 130(3.1)$ & $2 / 129(1.6)$ & $1 / 4(25.0)$ & $1 / 2(50.0)$ \\
\hline Week 4 & $18 / 130(13.9)$ & $19 / 128(14.8)$ & $4 / 18(22.2)$ & $1 / 19(5.3)$ \\
\hline Week 16 & $77 / 125(61.6)$ & $70 / 119(58.8)$ & $8 / 77(10.4)$ & $11 / 70(15.7)$ \\
\hline Week 20 & $86 / 122(70.5)$ & $80 / 119(67.2)$ & $6 / 86(7.0)$ & $11 / 80(13.8)$ \\
\hline Week 32 & $86 / 115(74.8)$ & $82 / 111(73.9)$ & $7 / 86(8.1)$ & $11 / 82(13.4)$ \\
\hline Week 52 & $82 / 118(69.5)$ & $84 / 114(73.7)$ & $14 / 82(17.1)$ & $18 / 84(21.4)$ \\
\hline
\end{tabular}

ADAs antidrug antibodies, NADAs neutralizing antidrug antibodies, $\mathrm{n} / \mathrm{N}$ number of positive patients/number of patients tested at each time point

than in real-world clinical practice. The absence of PASI 90 and PASI 100 evaluations are other potential limitations of this study. Furthermore, as plaque psoriasis is a chronic disease, the follow-up period of 4 weeks in this study is rather short, and thus long-term observation would be beneficial for monitoring the effectiveness and safety of HLX03.

\section{CONCLUSIONS}

This phase III study demonstrated that HLX03 was equivalent to adalimumab with regards to clinical efficacy, safety, and immunogenicity. These results add to the totality of evidence supporting HLX03 as an adalimumab biosimilar and confirm HLX03 as an effective and welltolerated treatment option for patients with moderate-to-severe plaque psoriasis in China.

\section{ACKNOWLEDGEMENTS}

The authors would also like to thank the patients who participated in this trial and their families.

Funding. This study was funded by Shanghai Henlius Biotech, Inc., Shanghai, China. The rapid service and open access fees were also sponsored by Shanghai Henlius Biotech, Inc.

Medical writing, editorial, and other assistance. Writing and editorial support for this manuscript was provided by Jean Turner, Xiaoguang $\mathrm{Xu}$, and Steve Dawber of Parexel, and Chen Hu of Shanghai Henlius Biotech, Inc. and funded by Shanghai Henlius Biotech, Inc. The authors would like to thank the principal investigators, clinicians, study coordinators, nurses, and the clinical study team (Clinical Operations: Jing Li, Liman Zhang; Medical R\&D: Jing Zhang; Statistics: Jiancheng Cheng, Wenting Qiu; Pharmacokinetics: Xiaodi Zhang; Medical Publication: Qiyu Luo and Shiqi Zhong), and Wenjie Zhang from Shanghai Henlius Biotech, Inc.

Authorship. All named authors meet the International Committee of Medical Journal Editors (ICMJE) criteria for authorship for this article, take responsibility for the integrity of the work as a whole, and have given their approval for this version to be published.

Author contributions. Jianzhong Zhang contributed to the study conception and design. Lin Cai, Linfeng Li, Hao Cheng, Yangfeng Ding, Zhenshu Biao, Shifa Zhang, Songmei Geng, Quanzhong Liu, Hong Fang, Zhiqi Song, Yan Lu, Shanshan Li, Qing Guo, Juan Tao, Li 
He, Jun Gu, Qinping Yang, Xiuping Han, Xinghua Gao, Danqi Deng, Shenqiu Li, and Qingyu Wang and Jun Zhu contributed to material preparation, data collection and analysis. All authors reviewed and provided comments on the manuscript, and approved the final submitted manuscript.

Disclosures. Qingyu Wang and Jun Zhu are employees of Shanghai Henlius Biotech, Inc. Lin Cai, Linfeng Li, Hao Cheng, Yangfeng Ding, Zhenshu Biao, Shifa Zhang, Songmei Geng, Quanzhong Liu, Hong Fang, Zhiqi Song, Yan Lu, Shanshan Li, Qing Guo, Juan Tao, Li He, Jun $\mathrm{Gu}$, Qinping Yang, Xiuping Han, Xinghua Gao, Danqi Deng, Shenqiu Li and Jianzhong Zhang have nothing to disclose.

Compliance with ethics guidelines. The study was conducted in accordance with the principles of both Good Clinical Practice from the International Council for Harmonization and the 1964 Declaration of Helsinki. The protocol and all amendments of this study were approved by the regulatory authorities and the ethics committees at all participating centers.

Data availability. Data are available on reasonable requests from the corresponding author.

Open Access. This article is licensed under a Creative Commons Attribution-NonCommercial 4.0 International License, which permits any non-commercial use, sharing, adaptation, distribution and reproduction in any medium or format, as long as you give appropriate credit to the original author(s) and the source, provide a link to the Creative Commons licence, and indicate if changes were made. The images or other third party material in this article are included in the article's Creative Commons licence, unless indicated otherwise in a credit line to the material. If material is not included in the article's Creative Commons licence and your intended use is not permitted by statutory regulation or exceeds the permitted use, you will need to obtain permission directly from the copyright holder. To view a copy of this licence, visit http://creativecommons.org/licenses/bync/4.0/.

\section{REFERENCES}

1. Ding X, Wang T, Shen Y, Wang X, Zhou C, Tian S, et al. Prevalence of psoriasis in China: a populationbased study in six cities. Eur J Dermatol. 2012;22(5): 663-7.

2. AbbVie Inc. Humira ${ }^{\circledR}$ prescribing information. https://www.rxabbvie.com/pdf/humira.pdf. Accessed 24 Aug 2020.

3. AbbVie Inc. Humira ${ }^{\circledR}$ Summary of product characteristics. 2020. https://www.ema.europa.eu/en/ documents/product-information/humira-eparproduct-information_en.pdf. Accessed 19 Aug 2020.

4. National Medical Products Administration China. NMPA guidelines for clinical trials of adalimumab injection biosimilars. 2020. https://www.nmpa.gov. cn/directory/web/nmpa/images/

1596531455261017949.pdf. Accessed 24 Aug 2020.

5. AbbVie Inc. Chinese-licensed adalimumab injection instructions. 2020. https://xiumeile.com/ index.php/shuomingshu.html. Accessed $24 \mathrm{Aug}$ 2020.

6. Zhao Y, Lai W. Patient considerations and targeted therapies in the management of psoriasis in Chinese patients: role of ustekinumab. Patient Prefer Adherence. 2014;8:865-72.

7. Puig L, López-Ferrer A. Biosimilars for the treatment of psoriasis. Expert Opin Biol Ther. 2019;19(10): 993-1000.

8. Kim H, Alten R, Avedano L, Dignass A, Gomollon F, Greveson $\mathrm{K}$, et al. The future of biosimilars: maximizing benefits across immune-mediated inflammatory diseases. Drugs. 2020;80(2):99-113.

9. National Medical Products Administration China. NMPA technical guidelines for development and evaluation of biosimilars. 2015. http://www.cde. org.cn/attachmentout.do?mothed=list\&id= 7b8967469153a13c. Accessed 24 Aug 2020.

10. European Medicines Agency. Guideline on similar biological medicinal products containing biotechnology-derived proteins as active substance: non-clinical and clinical issues. 2014. https://www. ema.europa.eu/en/documents/scientific-guideline/ guideline-similar-biological-medicinal-products- 
containing-biotechnology-derived-proteins-active_ en-2.pdf. Accessed 1 Sept 2020.

11. WHO Expert Committee on Biological Standardization. Guidelines on evaluation of similar biotherapeutic products (SBPs) 2009. https://www. who.int/biologicals/areas/biological_therapeutics/ BIOTHERAPEUTICS_FOR_WEB_22APRIL2010.pdf. Accessed 1 Sept 2020.

12. US Food and Drug Administration. Development of therapeutic protein biosimilars: comparative analytical assessment and other quality-related considerations 2019. https://www.fda.gov/media/ 125484/download. Accessed 1 Sept 2020.

13. Al-Sabbagh A, Olech E, McClellan JE, Kirchhoff CF. Development of biosimilars. Semin Arthritis Rheum. 2016;45(5 Suppl):S11-8.

14. China National Medical Products Administration. Adalimumab biosimilars approved in China. http:// app1.nmpa.gov.cn/data_nmpa/face3/base.

jsp?tableId=25\&tableName=TABLE25\&title=\%B9\% FA\%B2\%FA\%D2\%A9\%C6\%B7\&bcId= 152904713761213296322795806604. Accessed 11 Sept 2020.

15. Reynolds KA, Pithadia DJ, Lee EB, Liao W, Wu JJ. Safety and effectiveness of anti-tumor necrosis factor-alpha biosimilar agents in the treatment of psoriasis. Am J Clin Dermatol. 2020;21(4):483-91.

16. Tu L, Wei Q, Xie Y, Shi G, Liu H, Huang Q, et al. FRI0415 biosimilar Bat1406 versus adalimumab therapy on active ankylosing spondylitis: a randomized, double-blinded, multicenter, controlled phase 3 trial. Ann Rheum Dis. 2019;78(2):895.

17. Su J, Li M, He L, Zhao D, Wan W, Liu Y, et al. Comparison of the efficacy and safety of adalimumab (Humira) and the adalimumab biosimilar candidate (HS016) in Chinese patients with active ankylosing spondylitis: a multicenter, randomized, double-blind, parallel, phase III clinical trial. BioDrugs. 2020;34(3):381-93.

18. Xu H, Li Z, Wu J, Xing Q, Shi G, Li J, et al. IBI303, a biosimilar to adalimumab, for the treatment of patients with ankylosing spondylitis in China: a randomised, double-blind, phase 3 equivalence trial. Lancet Rheumatol. 2019;1(1):e35-43.

19. National Medical Products Administration China. Information release of the drug approval document pending receipt on December 07, 2020. 2020. https://www.nmpa.gov.cn/zwfw/sdxx/sdxxyp/ yppjfb/20201207163143123.html. Accessed 18 Dec 2020.

20. Zhang E, Xie L, Qin P, Lu L, Xu Y, Gao W, et al. Quality by design-based assessment for analytical similarity of adalimumab biosimilar HLX03 to Humira ${ }^{\circledR}$. AAPS J. 2020;22(3):69.

21. Sun J, Chen G, Wu M, Han Y, Gao H, Zhang T, et al. FRI0086 China-manufactured adalimumab biosimilar, HLX03, demonstrated pharmacokinetic equivalence and comparable safety to reference adalimumab. Ann Rheum Dis. 2019;78(2):706.

22. Papp K, Bachelez H, Costanzo A, Foley P, Gooderham M, Kaur P, et al. Clinical similarity of biosimilar ABP 501 to adalimumab in the treatment of patients with moderate to severe plaque psoriasis: a randomized, double-blind, multicenter, phase III study. J Am Acad Dermatol. 2017;76(6):1093-102.

23. Fiorino G, Girolomoni G, Lapadula G, Orlando A, Danese S, Olivieri I. The use of biosimilars in immune-mediated disease: a joint Italian Society of Rheumatology (SIR), Italian Society of Dermatology (SIDeMaST), and Italian Group of Inflammatory Bowel Disease (IG-IBD) position paper. Autoimmun Rev. 2014;13(7):751-5.

24. Gerdes S, Mrowietz U, Augustin M, von Ralph K, Enk A, Strömer K, et al. Biosimilars in dermatology—theory becomes reality. J Dtsch Dermatol Ges. 2018;16(2):150-60.

25. Markus R, McBride HJ, Ramchandani M, Chow V, Liu J, Mytych D, et al. A review of the totality of evidence supporting the development of the first adalimumab biosimilar ABP 501. Adv Ther. 2019;36(8):1833-50.

26. Committee on Psoriasis, Chinese Society of Dermatology. Guideline for the diagnosis and treatment of psoriasis in China (2018 simplified edition). Chin J Dermatol. 2019;52(4):223-30.

27. Cai L, Gu J, Zheng J, Zheng M, Wang G, Xi LY, et al. Efficacy and safety of adalimumab in Chinese patients with moderate-to-severe plaque psoriasis: results from a phase 3, randomized, placebo-controlled, double-blind study. J Eur Acad Dermatol Venereol. 2017;31(1):89-95.

28. Saurat JH, Stingl G, Dubertret L, Papp K, Langley RG, Ortonne JP, et al. Efficacy and safety results from the randomized controlled comparative study of adalimumab vs. methotrexate vs. placebo in patients with psoriasis (CHAMPION). Br J Dermatol. 2008;158(3):558-66.

29. Menter A, Tyring SK, Gordon K, Kimball AB, Leonardi CL, Langley RG, et al. Adalimumab therapy for moderate to severe psoriasis: a randomized, controlled phase III trial. J Am Acad Dermatol. 2008;58(1):106-15.

30. Asahina A, Nakagawa H, Etoh T, Ohtsuki M, Adalimumab MSG. Adalimumab in Japanese patients 
with moderate to severe chronic plaque psoriasis: efficacy and safety results from a phase II/III randomized controlled study. J Dermatol. 2010;37(4): 299-310.

31. Menter A, Thaci D, Wu JJ, Abramovits W, Kerdel F, Arikan D, et al. Long-term safety and effectiveness of adalimumab for moderate to severe psoriasis: results from 7-year interim analysis of the ESPRIT registry. Dermatol Ther (Heidelb). 2017;7(3): 365-81.

32. Mansouri Y, Goldenberg G. Biologic safety in psoriasis: review of long-term safety data. J Clin Aesthet Dermatol. 2015;8(2):30-42.

33. Strand V, Gonçalves J, Hickling TP, Jones HE, Marshall L, Isaacs JD. Immunogenicity of biosimilars for rheumatic diseases, plaque psoriasis, and inflammatory bowel disease: a review from clinical trials and regulatory documents. BioDrugs. 2020;34(1): 27-37.
34. Hercogová J, Papp KA, Chyrok V, Ullmann M, Vlachos P, Edwards CJ. AURIEL-PsO: a randomized, double-blind phase III equivalence trial to demonstrate the clinical similarity of the proposed biosimilar MSB11022 to reference adalimumab in patients with moderate-to-severe chronic plaquetype psoriasis. Br J Dermatol. 2020;182(2):316-26.

35. Gorovits B, Baltrukonis DJ, Bhattacharya I, Birchler MA, Finco D, Sikkema D, et al. Immunoassay methods used in clinical studies for the detection of anti-drug antibodies to adalimumab and infliximab. Clin Exp Immunol. 2018;192(3):348-65.

36. Chiu HY, Wang TS, Chan CC, Lin SJ, Tsai TF. Risk factor analysis for the immunogenicity of adalimumab associated with decreased clinical response in chinese patients with psoriasis. Acta Derm Venereol. 2015;95(6):711-6. 